I.

Aus der chirurgischen Klinik (Direktor Prof. Dr. Madelung) und dem pathologischen Institut (Direktor Prof. Dr. Chiari) zu Strassburg i. E.

\title{
Beitrag zur Kenntnis der Luxatio genu congenita anterior.
}

\author{
Von \\ Dr. Jung, Assistent der chirurg. Klinik.
}

Mit 5 Abbildungen im Text.

In einer 1900 erschienenen Arbeit stellte Drehmann ${ }^{1}$ ) die bis dahin bekannt gewordenen Fälle angeborener Kniegelenksluxation zusammen. Er sichtete das vorhandene Material und beseitigte die Verwirrung, welche bezüglich der Nomenklatur der angeborenen Kniedeformitäten in der Literatur herrschte, indem er den Begriff der Luxation nach vorn gegenüber dem Genu recurvatum feststellte. Das Genu recurvatum könne zwar in die Luxation übergehen; im anatomischen Sinne als Genu recurvatum seien nur die Fälle anzusehen, in denen bei normaler Beugefähigkeit eine abnorme Steigerung der Extension möglich sei; für alle die Fälle aber, bei denen die Beugefähigkeit beschränkt und die Kondylen des Femur nach hinten abgewichen seien, empfahl er die Bezeichnung kongenitale Knieluxation (nach vorn); die verschiedenen Erscheinungsformen dieser Deformität seien nur als verschiedene Stadien zu unterscheiden.

Bei der Besprechung der pathologischen Anatomie der kongenitalen Knieluxation stützte sich Drehmann auf 16 publizierte Autopsien, über eine weitere Autopsie berichtete seitdem noch Delanglade2). Alle diese beschriebenen Präparate stammen aber von Neugeborenen oder Föten. Über die anatomischen Verhältnisse der Luxation bei älteren Kindern erhielt man Aufschluss teilweise durch Röntgenbilder (Drehmann) teilweise durch Beobachtungen, die gelegentlich operativer Eingriffe gemacht wurden. (Reiner ${ }^{3}$ ), Bacilierit)).

1) Zeitschrift f. orthopäd. Chirurgie. Bd. 1900.

2) Revue d'orthopédie. 1903. Nr. 3.

$\left.{ }^{3}\right)$ Zeitschrift f. orthopäd. Chirurgie. Bd. XIII.

4) Archiv f. Orthopädie, Mechanotherapie etc. Bd. III.

Arch. f, Orthop., Mechanoth. u. Unf.-Chir. XI, 1. 
Da ich Gelegenheit hatte, bei einem 14 Monate alten Mädchen die Deformität zu studieren, sei es mir gestattet, über den anatomischen B efund dieses Falles zu berichten. Auf die klinischen Symptome dieser Missbildung und ihre Behandlung will ich nicht näher eingehen; ich verweise hierfür auf die neueren Monographien von Drehmann, Bacilieri und Wehsarg ${ }^{1}$, sowie auf die Lehrbücher von Joachimsthal und Hoffa und den Vortrag von Perthes auf dem Orthopädenkongress von 1905.

Krankengeschichte:

Das $13 \frac{1}{2}$ Monate alte Mädchen stammt aus einer Familie, in welcher von Missbildungen nichts bekannt ist, und wurde nach normal verlaufener Schwangerschaft normalerweise geboren. Gleich bei der Geburt fiel den Eltern eine "Verkrümmung der Füsse" des Kindes auf; sie achteten jedoch nicht darauf. Erst da im Laufe des letzten Monats angestellte Gehversuche dem Kinde Schwierigkeiten machten, suchten sie am 22. I. 10. die chirurgische Klinik auf.

Pastös aussehendes, gut genährtes Kind. Gesichtsfarbe zyanotisch. Caput quadratum; starke Prominenz der Frontal- und Parietalböcker. Grösster Kopfumfang $45 \mathrm{~cm}$, grosse Fontanelle etwa $3 \mathrm{qcm}$ gross.

Keine wesentliche Auftreibung der Röhrenknochen- und Rippenepiphysen.

Beide Hände stehen in leichter Subluxationsstellung volar- und ulnarwärts. Es besteht ausserordentliche Schlaffheit sämtlicher Fingergelenke, hauptsächlich der Metakarpo-phalangealgelenke, so dass eine passive Überstreckung bis fast $270^{\circ}$ möglich ist. Der linke Mittelfinger erscheint $\mathrm{um}^{1 / 2} \mathrm{~cm}$ kürzer als die benachbarten Finger; die Verkürzung beruht auf einer Verkürzung des III. Metakarpus.

Beide Hüftgelenke sind normal.

Beide Füsse stehen in ausgesprochener Kalkaneovalgusstellung. Die Abduktion des Metatarsus ist links etwas stärker als rechts. Die Halluzes stehen in fast rechtwinkliger Valgusstellung; auch hier besteht grosse Schlaffheit sämtlicher Gelenke.

Rechter Unterschenkel etwas abduziert (Genu valgum).

Linker Oberschenkel erscheint etwas magerer als der rechte. Linker Unterschenkel liegt in der Ruhe auf der Unterlage in Valgusstellung von etwa $150^{\circ}$, ist gegen den Oberschenkel um fast $90^{\circ}$ auswärts rotiert, so dass seine Aussenfläche die Unterlage berührt. Beim Anheben des Fusses geht der Unterschenkel mit, während der Oberschenkel auf der Unterlage liegen bleibt. Es kann das Knie um $40^{\circ}$ überstreckt werden; dabei treten in der Haut oberhalb des Gelenkes mehrere quere Falten auf. Passive Flexion nur um $50^{\circ}$ möglich.

Palpation liess annehmen, dass die linke Patella fehlt; die Tibia ist nach vorn und aussen gegen den Oberschenkel fast völlig luxiert, so dass man von der Kniekehle aus den Condylus fem-int. und einen grossen Teil des Cond. ext. abtasten kann.

1) Archiv f. Orthop., Mechanoth. etc. Bd. III. 
Schon bei der Aufnahme wurde bei dem Kinde eine diffuse akute Bronchitis festgestellt; es gesellte sich bald dazu eine Pneumonie des linken Unterlappens, dann des rechten Oberlappens; das schwächliche Kind erlag.

Die von Prof. $\mathrm{Ch}$ iari am 22. II. 10. vorgenommene Sektion ergab als Todesursache: Eitrige Pneumonie des linken Unterlappens, Abszess im rechten Oberlappen. Zeichen von Rachitis wurden nicht gefunden.

Während aus äusseren Gründen die Füsse nicht seziert wurden, konnten beide Knie mit den zugehörigen Muskeln unter der Haut ausgeschält und konserviert werden.

$$
\text { Beschreibung des Präparats. }
$$

Das rechte Knie bot ausser geringer Valgusstellung nichts Besonderes, konnte daher bei der Untersuchung des linken Knies als normal zum Vergleich herangezogen werden.

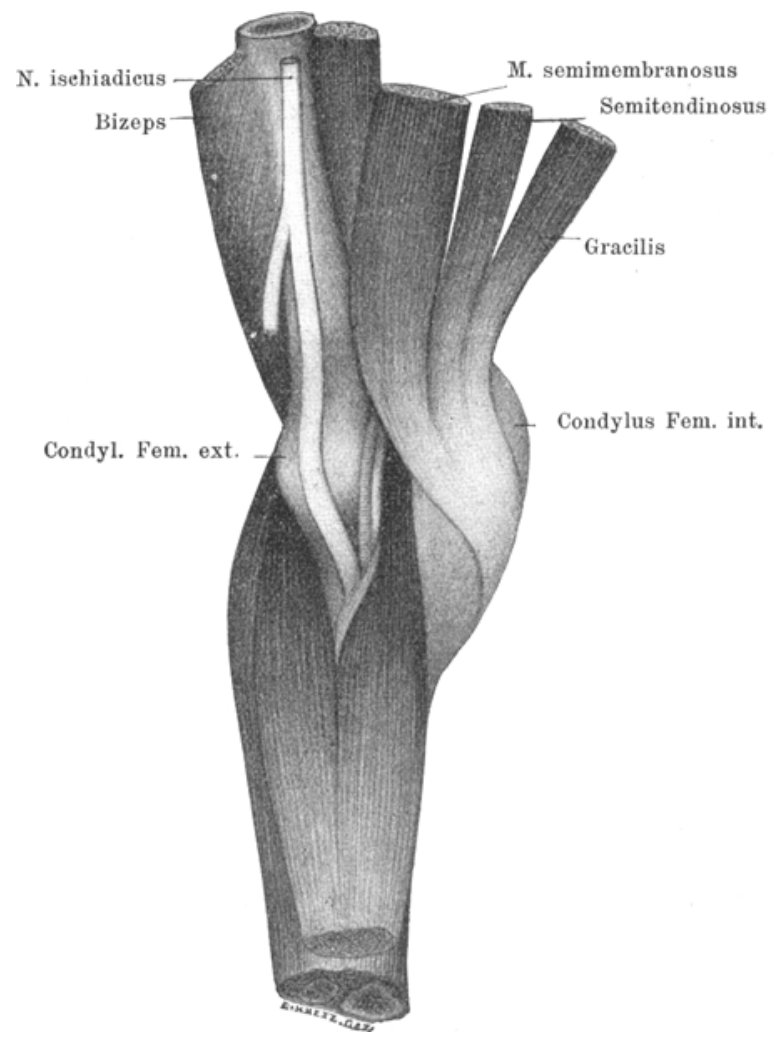

Fig. 1.

Schon bei Besichtigung des rohen Präparats kann eine geringere Entwicklung der ganzen linken unteren Extremität im Vergleich zur rechten festgestellt werden. Die Knochen sind um ein Drittel weniger dick als rechts.

Bei der Präparation der Muskeln (cf. Fig. I.) findet sich, dass der Quadrizeps in toto auffallend schwach und blass ist; kräftiger sind die 
Beuger entwickelt. Die Sehne des Bizeps femoris verläuft vor dem Cond. Fem. ext. nach der Aussenseite der Tibia. Die Mm. semitendinosus, semimembranosus und gracilis verlaufen hinter dem Cond. fem. int. nach ihrem Ansatz an der Innenseite der Tibia. Der mediale Kopf des Gastroknemius ist nach aussen verschoben und lässt den Cond. tib. med. frei. Während die Gefässe in der Tiefe der Kniekehle liegen, ist der N. tibialis über die Kuppe des Cond. ext. nach aussen verlagert.

Nach Entfernung der Muskeln lässt sich die Stellung des Femur zu den Unterschenkelknochen genauer übersehen (cf. Fig. II.-IV.). Zunächst ist zu

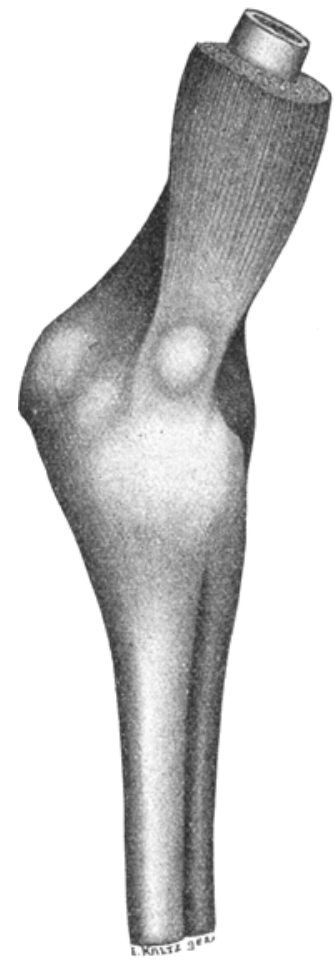

Fig. 2.

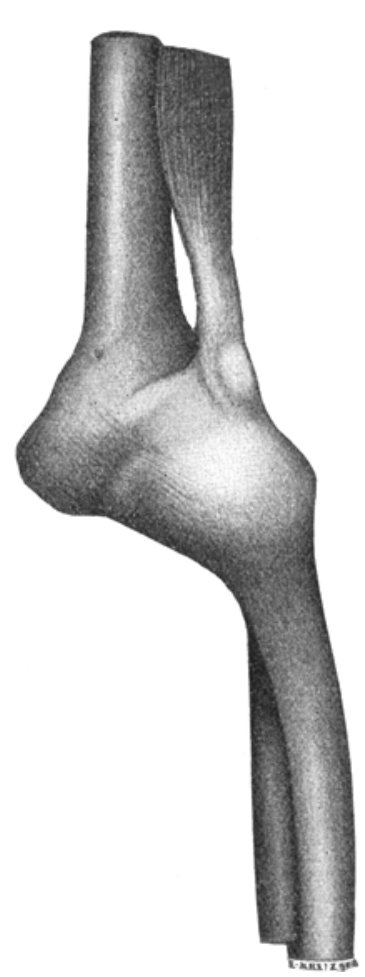

Fig. 3.

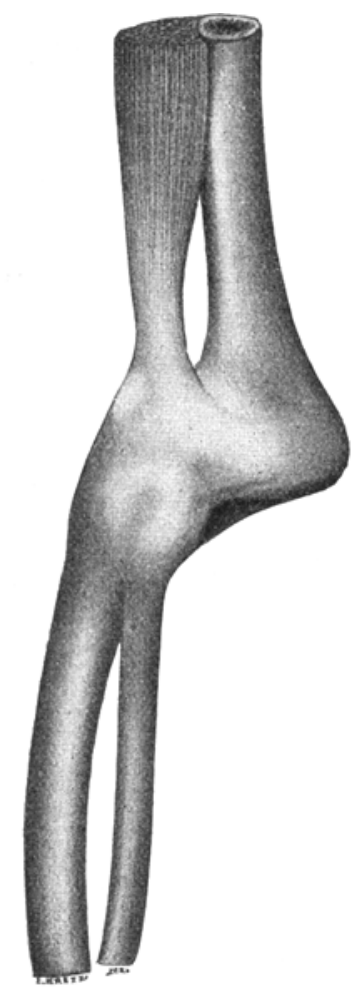

Fig. 4.

erwähnen, dass die Patella, die am Lebenden wie gesagt, nicht gefühlt werden konnte, dennoch vorhanden ist; sie ist kaum $1 / 3$ so gross als die rechte und liegt nicht dem Femur an, sondern ist mit der Quadrizepssehne in ein reichliches, zwischen Femur und Haut liegendes Fettpolster, eingebettet.

Der Unterschenkel bildet mit dem Oberschenkel einen nach aussen offenen Winkel von $145^{\circ}$. Von den Femurcondylen sind die hintere und untere Fläche, vom äusseren sogar ein kleiner Teil der Vorderfläche ausser jedem Kontakt mit der Tibia; diese artikuliert nur mit der Vorderfläche der Femurkondylen. Das obere Drittel der Tibia ist bogenförmig nach hinten verkrümmt. 
Die Gelenkkapsel ist infolge der Konservierung in Alkohol stark geschrumpft; man kann trotzdem noch erkennen, dass während sie auf der Hinterseite des Gelenks gespannt war, sie in den vorderen und seitlichen Partien schlaffer gewesen sein muss. Ihr Ansatz ist hinten am Femur etwas tiefer als normal, so dass ein kleiner Teil der Fossa intercondylica noch ausserhalb des Gelenks steht. Der Recessus sup. ist nur als schlaffe Falte angedeutet. Von den Verstärkungsbändern sind entsprechend der geringen Entwickelung der Patella die Ligg. alaria nicht darzustellen; das Lig. collaterale tibiale ist gut entwickelt; das Lig. coll. fib. schwächer, verläuft entsprechend der Verschiebung des Unterschenkeìs an die Vorderfläche des Femur nahezu horizontal.

Nach Eröffnung der Gelenkkapsel zeigt sich um so dentlicher, dass die Tibia nur mit der Vorderfläche der Femurkondylen artikuliert. Die Menisci sind als kleine flache Scheiben vorhanden, das Lig. mucosum sehr stark entwickelt; die Ligg. cruciata sind richtig inseriert, gut entwickelt (kräftiger als am rechten Knie), etwas ausgezogen, in der Nähe ihres Ansatzes an der Eminentia intercondylica tibiae von zahlreichen, feinen roten Punkten durchsetzt; diese Punkte sind wohl als kleine Hämorrhagien zu deuten, entstanden infolge des starken Zuges, dem die Ligg. cruciata ausgesetzt waren.

Der Knorpelüberzug der Femurepiphyse ist nicht glatt, sondern an zahlreichen Stellen atrophisch. Während die Vorderfläche des Gelenkteiles eines normalen Femur glatt ist oder zwischen den Kondylen nur eine ganz seichte Einsenkung aufweist, befindet sich hier an der Vorderfläche eine deutliche Fovea intercondylica; im Grunde dieser Grube ist der Knorpel aufgefasert.

Einige Punkte des anatomischen Befundes verdienen eine besondere Besprechung.

Zunächst das Verhalten der Patella. Trotz mehrfacher sorgfältiger Untersuchung am Lebenden wurde die Patella nicht gefühlt. Sie fand sich dann aber doch bei der Autopsie, allerdings sehr klein und ausser Kontakt mit dem Femur.

Viele Beobachter haben darauf aufmerksam gemacht, dass bei der uns beschäftigenden Kniedeformität häufig die Patella ursprïnglich vermisst, bei einer späteren Untersuchung oder bei der Sektion doch aber gefunden wurde. Autoptisch wurde der vollständige Defekt der Patella bei der angeborenen Luxation des Kniees nach vorn noch nicht festgestellt. Von Rosenfeld ${ }^{1}$ ) wird ein 1 jähriges Mädchen erwähnt, das wegen Genu recurvatum behandelt wurde; hier wurde der vollständige Mangel der Patella nach dem Röntgenogramm angenommen. Dazu möchte ich bemerken, dass durch das bei meinem Fall am Lebenden angefertigte Röntgenbild die Patella auch nicht dargestellt wurde, jedenfalls deshalb, weil das in der Entwickelung zurückgebliebene Gebilde noch nicht verknöchert war, und das kleine Knorpelstück sich von den Weichteilen im Röntgenbilde nicht differenzieren liess.

1) Zeitschrift f. orthopäd. Chirurgie. 1902. Bd. X. 
Jang,

Die geringe Entwickelung der Patella bei den meisten Fällen von angeborener präfemoraler Knieluxation erklärt sich aus dem mangelnden Kontakt mit dem Femur und der herabgesetzten, oft gänzlich fehlenden Funktion des Quadrizeps. Denn nach Brunner') verdankt die Patella, die genetisch in keinem Zusammenhang mit den Unterschenkelknochen stehend, sich später differenziert als diese, als Sesambein ihre Entwickelung der Reizung der gut funktionierenden Quadrizepssehne auf der präformierten Knorpelunterlage der Femurepiphyse.

Was das Verhalten der Muskulatur in meinem Falle betrifft, so ist die erwähnte Verkürzung des Quadrizeps, die Verlagerung des Bizeps nach vorn, der somit statt als Beuger als Überstrecker wirkte, sowie die Verlagerung der Semimuskeln nach hinten eine bei der kongenitalen Knieluxation nach vorn regelmässig beobachtete Erscheinung. Seltener scheint die bei meinem Präparat bemerkte (auf Fig. I sichtbare) Verlagerung des Gestroknemiusursprunges nach aussen $\mathrm{zu}$ sein; wenigstens ist sie bis jetzt nur einmal, von Bacilieri, beschrieben worden. Bacilieri fasst diese Abnormität im Verlaufe des Gastroknemius als Anpassung an die durch die Valgusstellung bedingte Achsenanomalie des Unterschenkels, als Abweichung im Sinne der Abduktion auf und setzt sie damit auf die gleiche Stufe wie die Verkürzung der Semimuskeln.

Besonderes Interesse verdient die erwähnte Verkrümmung der Tibia nach hinten. Von Delanglade wurde bei einem neugeborenen Mädchen mit Knieluxation eine Verschiebung in der Dia-Epiphysenachse des unteren Femurendes mit Epiphysiolyse beobachtet; die untere Femurepiphyse bildete mit dem Femurschaft einen nach vorn offenen, stumpfen Winkel. Einen ähnlichen Befund machte $\left.\mathrm{Barth}{ }^{2}\right)$; auch bei seinem Falle stand die Linie des Fpiphysenansatzes zu der Achse des Oberschenkels nicht in einem rechten, sondern in einem ungefäbr ${ }^{2} / 3$ rechten, nach vorn offenen Winkel. K i r m is s o ${ }^{3}$ ) der Delanglade's Fall bespricht, sieht in der Verschiebung der Epi- zur Diaphyse des Femur die Folge eines lange einwirkenden abnormen Druckes infolge der anhaltenden, fehlerhaften intrauterinen Lage des Fötus und stützt diese Annahme einer lange anhaltenden Ursache für die Verkrümmung durch den histologischen Befund von Störungen in der Ossifikation, den Delanglade erhob.

Das bei meinem Präparat angefertigte Röntgenbild (cf. Fig. 5.) zeigt, dass von einer Epiphysenlösung am unteren Femurende oder speziell am oberen Tibia ende keine Rede sein kann. Die Epiphysenlinie der Tibia steht senkrecht auf der Achse des Knochens; der Knochenkern der Epiphyse steht genau in der Fortsetzung der verknöcherten Diaphyse. Die Verkrümmung, die schon im oberen Drittel des Tibiaschaftes angedeutet ist, betrifft hauptsächlich den knorpeligen Teil der Epiphyse. Aus der richtigen Lage des Knochenkerns geht wohl hervor, dass die starke Verkrümmung des Tibia-

1) Virchows Archiv. Bd. 122.

$\left.{ }^{2}\right)$ Archiv f. klin. Chirurgie. Bd. 31.

3) Revue d'orthopédie. 1903. Nr. 5. 
kopfes nicht die Folge einer Epiphysenlösung gewesen und jedenfalls zu einer Zeit, wo der Epiphysenkern bereits angelegt war, also nach der Geburt, entstanden ist. Ich nehme an, dass die "Retroversion" des knorpeligen Teils der Tibiaepiphyse eine Folge der Belastung ist bei den Gehversuchen, die das Kind ausführte. Eine weitere Verschiebung der Tibia aufwärts an der Vorderfläche des Femur, wie sie sich als Folge der Belastung hätte einstellen können und wie sie als Endstadium der kongenitalen Knieluxation beschrieben wird, wurde durch die Ligg-cruciata verhindert, die bei meinem Präparat im Gegensatz zu den meisten anderen Beobachtungen relativ derb und nur wenig veriängert waren.

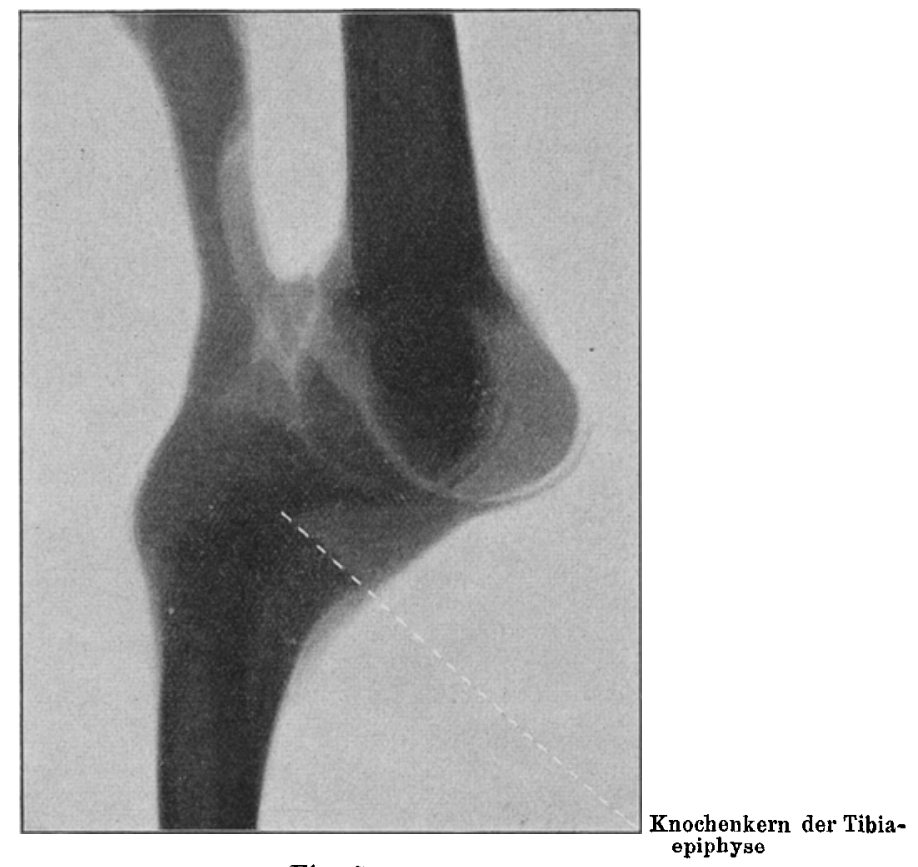

Fig. 5 .

Von praktischer Becleutung ist eine derartige Verkrümmung der Tibia insofern, als sie besonders wenn die Behandlung erst spät, nach Vollendung der Ossifikation einsetzt, den orthopädischen Erfolg einer operativen Reposition des-luxierten Gelenkes beeinträchtigen kann.

Die Frage der Ätiologie der angeborenen Knieluxation ist noch in Dunkel gehüllt. Ich will auf die verschiedenen Hypothesen nicht näher eingehen. Der Umstand, dass ausser der einseitigen Knieluxation bei meinem Falle noch Valgusstellung der Hände, Kalkaneovalgusstellung der Füsse beobachtet wurden, wird die Annahme einer abnormen intrauterinen Belastung rechtfertigen, sei es dass, wie $\mathrm{M} \ddot{\text { ll }}$ er ${ }^{1}$ ) annimmt, durch Mangel an Fruchtwasser ein abnormer Druck der Uteruswand auf die Extremitäten einwirkte, oder dass nach Drehmann aus irgend einer Ursache während des intra-

1) Arbeiten aus der Leipziger Poliklinik. I.-II. 1888-1892. 
uterinen Lebens die Extremitäten in eine Zwangslage gebracht und festgehalten wurden. Anamnestisch liess sich in unserem Fall weder von der Mutter noch von der Hebamme irgend eine bei der Geburt beobachtete Abnormität (in der Menge des Fruchtwassers oder in der Haltung des Kindes) feststellen.

Mit der Annahme einer abnormen intrauterinen Belastung ist aber noch nicht die erwähnte Schlaffheit sämtlicher Finger- und Zehengelenke erklärt. Dieser Befund legt den auch von Bacilieri bei einem ähnlichen Falle ausgesprochenen Gedanken nahe, dass vielleicht durch eine primäre fötale Erkrankung multipler Gelenke Ernährungsstörungen, dann abnorme Schlaffheit ihrer Kapseln bedingt wurden. Da wir jedoch über fötale Erkrankungen überhaupt noch sehr wenig wissen, kommen wir hier über eine Vermutung nicht hinaus. 